\title{
MANAGEMENT OF SUPRAGASTRIC BELCHING WITH COGNITIVE BEHAVIOURAL THERAPY: FACTORS DETERMINING SUCCESS AND FOLLOW-UP OUTOCOMES AT 6-12 MONTHS POST THERAPY
}

Short Title: Supragastric belching and CBT

Akinari Sawada ${ }^{1}$, Natasha Anastasi ${ }^{2}$, Alicia Green ${ }^{1}$, Esteban Glasinovic ${ }^{1}$, Emily Wynter ${ }^{1}$, Ahmed Albusoda ${ }^{1,}$ Peter Hajek ${ }^{2}$, Daniel Sifrim ${ }^{1}$

1. Wingate Institute of Neurogastroenterology, Blizard Institute, Barts and The London School of Medicine and Dentistry, Queen Mary University of London, UK

2. Health and Lifestyle Research Unit, Wolfson Institute of Preventive Medicine, Queen Mary, University of London, UK

Corresponding Author: Daniel Sifrim MD, PhD

Barts and The London School of Medicine and Dentistry Queen Mary, University of London, UK. Upper GI Physiology Unit Royal London Hospital

Address: Wingate Institute of Neurogastroenterology. 26 Ashfield Street, London E12AJ, UK

\section{Acknowledgements:}

Grant support - The study was conducted with financial support from Research Grant DDCH1A3R, Queen Mary University of London

Disclosures: Daniel Sifrim receives research grants from Reckitt Benckiser UK, Jinshan Technology China and Alfa Sigma, Italy. The remaining authors declare no conflicts of interest with this study.

Guarantor of the article: Daniel Sifrim MD, PhD

\section{Specific author contributions:}

DS, AS and PH: study concept and design of study protocol. NA, AG, EG, EW, recruited, treated patients and analysed impedance-pH studies. AS, AA and DS interpreted results of studies, prepared figures and drafted the manuscript. $\mathrm{PH}$ edited and revised the manuscript with final approval from DS. 


\section{Submitted in Abstract Form to 2019 DDW.}

Word count: 3106 (not including References, Tables and Legends)

\section{Summary}

Background: Supragastric belching (SGB) has a significant behavioural component. We recently used cognitive behavioural therapy (CBT) to treat SGB. We demonstrated that CBT significantly reduces symptoms and improves quality of life in $50 \%$ of patients that completed treatment.

Aims: To investigate factors associated to successful CBT for SGB and to assess symptoms 6-12 months after completion of CBT.

Methods: Records of 39 patients who had completed the CBT protocol were analysed. Percent pre-to-post treatment change in symptoms was assessed using a Visual Analogue Scale (VAS) score. We evaluated the association between "pre-treatment" factors and "during treatment" factors, and symptomatic outcomes. Symptoms were also assessed 6-12 months after treatment.

Results: From "pre-treatment factors", a lower number of SGBs $(P<0.01)$ and lower hypervigilance score $(P<0.04)$ were significantly associated with better outcome. From "during treatment factors" a higher CBT "proficiency score" [a) acceptance of the explanation that SGB is a behavioural phenomenon b) detection of a warning signal before belching $\mathrm{c}$ ) adherence to the exercises treatment] was associated with better outcome $(P=0.001)$. Multiple regression analysis found that number of SGBs, hypervigilance score and CBT proficiency 
score were independently associated with outcome $(P<0.01, P=0.01, P<0.01)$. VAS score before CBT $(267 \pm 79)$ decreased to $151 \pm 88$ soon after CBT $(P<0.001)$, and the effect persisted at 6-12 months follow up $(153 \pm 82)$.

Conclusions: Lower number of SGBs, lower hypervigilance score and higher patient's proficiency during CBT were associated with better CBT outcome. CBT positive effect lasted at least 6-12 months post-treatment.

Key words: supragastric belching, cognitive behavioural therapy, hypervigilance 


\section{Introduction}

Impedance-pH monitoring can detect gastric belching and supragastric belching (SGB). Gastric belching is a physiological mechanism to vent swallowed air from the stomach whereas SGB involves an intake of air from the mouth/pharynx into the oesophagus, followed immediately by expelling the oesophageal air using abdominal straining ${ }^{1}$.

SGB often accompanies gastroesophageal reflux disease (GERD), functional dyspepsia and/or irritable bowel syndrome (IBS) ${ }^{2-6}$. In some patients, SGB can induce gastroesophageal reflux ${ }^{3,4}$. Excessive SGB has a profound impact on health-related quality of life 7,8 .

Pharmacological therapy for SGB includes use of Baclofen and Pregabalin. These drugs can partially decrease the number of SGBs ${ }^{9,10}$, but their central side effects might preclude their chronic use. Previous studies have shown that cognitive behavioural therapy (CBT) can improve SGB ${ }^{8,11-16}$. Recently, our group reported that CBT significantly reduces the number of SGBs and improves quality of life. Our 10 weeks CBT protocol consisted of explaining the possible mechanism of SGB, raising awareness of the physical initial warning signals preceding belching, and teaching patients abdominal breathing manoeuvres and a mouth/tongue position to prevent it ${ }^{8}$. Half of our patients had a positive clinical response to CBT. Unlike pharmacotherapy, CBT does not cause side effects and its effect can last more than 6 months. On the other hand, CBT requires specialized clinical psychologists, a relative prolonged treatment and good patient's compliance. Furthermore, such treatment is not readily available in many institutions worldwide. It could be therefore, clinically relevant and useful to be 
able to identify, beforehand, those patients that are more likely to respond to CBT.

The aim of this study was to investigate factors contributing to successful CBT for SGB and to audit subjective outcomes at 6-12 months after completion of CBT.

\section{Materials and Methods}

Subjects

Fifty-one consecutive patients $[51 \%$ female, mean age 45 (20-72)] with pathological SGB (>13SGBs/24h) diagnosed by multichannel intraluminal impedance-pH monitoring (Sandhill Scientific, Inc, Highlands Ranch, CO, USA) were recruited at the Upper gastrointestinal Physiology Unit of the Royal London Hospital ${ }^{8}$. Out of 51 patients referred to the CBT protocol, 12 patients did not complete the treatment. The remaining 39 patients who completed the 10 -week CBT protocol were included in the current retrospective analysis. All of them had evaluation before and after treatment using a Visual analogue scale (VAS) for SGB symptoms severity (14), the Short Form Health Survey (SF-36), Big Five Inventory-10, and The Somatosensory Amplification Scale (Hypervigilance score). Thirty-one patients underwent a second impedance-pH monitoring after CBT.

\section{Subjective evaluation}

Visual analog scale (VAS)

A four-item VAS, measured subjective changes in the severity of belching symptom throughout the intervention ${ }^{14}$. Patients rated the following items from 0 (absence of symptoms) to 100 (very severe symptoms) as follows; Q1. How 
bothered are you by your belching? Q2. How much can you control your belching? Q3. How much are your daily activities affected by your belching? Q4. How much are your social activities affected by your belching?

VAS was recorded at $1^{\text {st }}$ (Pre CBT), $3^{\text {rd }}, 4^{\text {th }}$, and $5^{\text {th }}$ (Post CBT) CBT sessions. Evaluation of VAS score was repeated 6 to 12 months after the end of CBT, through a telephone interview.

Health related quality of life and personality assessment Health-related quality of life (HR-QOL) was assessed using the Short Form Health Survey (SF-36), which comprises eight domains as follows; physical functioning (daily life limitations due to health problems), bodily pain, limitations in fulfilling physical roles, general health and four psychological subscales of limitations in fulfilling emotional roles, vitality (energy/fatigue), social functioning and mental health. The scores of eight dimensions listed above were calculated using a dedicated algorithm ranging from 0 (where the respondent has the worst possible health) to 100 (where the respondent is in the best possible health) ${ }^{17}$. The Big Five Inventory-10, a short version of the Big Five Inventory, was used to evaluate each patient's personality. The dimensions of extraversion, agreeableness, conscientiousness, neuroticism, and openness were evaluated by 10 items with 5 -point scale from 1 (disagree strongly) to 5 (agree strongly) ${ }^{18}$.

Hypervigilance score

The Somatosensory Amplification Scale, a 10-item questionnaire, assesses hypervigilance to bodily sensations and the tendency to experience normal 
somatic sensations as uncomfortable or troubling ${ }^{19}$. The items are rated on a 5point scale from 1 (Not at all true) to 5 (Extremely true) and ratings are summed to produce a total amplification score that ranges from 10 to $50^{20}$, with average Somatosensory Amplification Scale scores between 24 and 29 21; scores higher than 30 may indicate increased somatization ${ }^{22}$.

Objective evaluation

Impedance-pH was performed "off" PPI before and after CBT. Tracings were manually analysed for presence of acid/non-acid reflux and SGBs. SGBs were identified as previously described ${ }^{1}$. In brief, a rapid rise in impedance $(\geq 1000 \Omega)$ moving in an aboral direction, followed by a return to baseline moving in the opposite direction. The total number of SGBs, acid and non-acid reflux episodes, and oesophageal acid exposure time (\%) were calculated.

\section{Cognitive Behavioural Therapy}

The CBT program consisted of 5 sessions during 10 weeks. There was an initial assessment by a gastroenterologist and a psychologist that was followed by four sessions of $\mathrm{CBT}$ as previously describe ${ }^{8}$.

CBT can be divided into a cognitive and a behavioural component. The cognitive component helps patients to understand the subconscious abnormal behaviour underlying SGB, to learn how the CBT exercise mechanism can prevent SGB, and to recognize the triggering events (warning signal) preceding SGB. Most patients detect a physical tension in the epigastrium and/or chest as a warning signal. During the behavioural component, the therapist teaches the patients two 
treatment exercises as follows; (1) slow diaphragmatic breathing with inhaling for $3 \mathrm{~s}$ and exhaling for $3 \mathrm{~s}$, and (2) mouth opening/tongue position i.e. patients are asked to breathe through their mouth slightly open with their tongue touching the back of the top front teeth. They are required to practice these exercises at least twice per day for 3-5 min in a supine or sitting position until they get familiar and confident with the techniques. They are instructed to use these manoeuvres to prevent SGB as often as possible, especially when they feel the warning sign.

\section{Patient's proficiency in CBT}

A proficiency score for CBT was developed to assess the patient's understanding and implementation of the CBT exercises. The Proficiency Score (from 0 to 5) was assessed by CBT trained psychologists (EW and NA) and included the following items:

a) Acceptance of the explanation that SGB is a behavioural phenomenon rather than an organic disease and understanding of the working mechanism of the exercises prescribed. This item was assessed at the end of the treatment $(0=$ nil acceptance of SGB diagnosis and seeking further reasons to explain their belching or GI symptoms, 1= open to SGB diagnosis and completing treatment, yet some uncertainty/hesitation reported, 2= full acceptance of SGB diagnosis and treatment.

b) The capacity of detection of a warning signal before belching was asked at the first CBT session. $0=$ no detection of any warning signals. $1=$ any warning signals were detected.

c) Adherence to the exercise preventing SGBs reflected whether the patient 
completed the behavioural exercises between sessions (i.e. whether they used the diaphragmatic breathing to prevent and stop belching as instructed) and used correctly in the prescribed way. This was based on the patient's self-report to the psychologist; $0=$ no adherence to treatment instructions meaning a patient did not use the treatment instructions to prevent belching, $1=$ mostly adhered to treatment instructions with some difficulty with frequency or accuracy of the technique, 2= adhered to treatment instructions as instructed and with appropriate technique.

\section{Statistical analysis}

Data are expressed as median (interquartile range) or mean $\pm \mathrm{SD}$. The severity of belching symptoms was measured using VAS score in pre-CBT, during CBT sessions and in post-CBT. The aim of the analysis was to examine if there were factors associated with the change in the severity of belching symptoms from pre to post-treatment. This was analysed as a continuous score and was found to have an approximately normal distribution.

Analyses examining factors associated with this outcome were performed in two stages. Firstly, the separate (univariable) association between each factor and the outcome was examined using linear regression. In the analysis for the association between CBT measurements and the percentage change in VAS score, each of the individual CBT components was assessed, along with the proficiency score for CBT. Patients with responses of zero for the acceptance and adherence components, were grouped with a score of 1 for analysis. The 
proficiency score was analysed as a continuous measurement and also split into categories. Subsequently, the joint association between the factors and outcome was assessed by multiple regression. To limit the number of factors in this stage of the analysis, only factors showing some association with the outcome in the univariable analyses $(p<0.2)$ were included. A backwards selection procedure was performed to retain only the significant factors in the final model.

Repeated measures ANOVA with Bonferroni post-hoc test was used to compare the VAS score during CBT sessions and among pre-CBT, post-CBT, and 6-12 months after CBT. $\mathrm{P}<0.05$ was considered statistically significant. 


\section{Results}

Pre-treatment factors associated to CBT outcome

\section{Demographic and clinical data}

Out of 39 patients who completed the CBT session, 12 patients (31\%) took standard dose of PPIs and 14 patients (36\%) took double dose of PPIs at diagnosis. Twenty-three patients (59\%) were native English speaker. Nine Patients $(23 \%)$ had education to degree or above. Seventeen patients $(44 \%)$ complained of belching as primary symptom and the duration of belching symptom was 2.5 years (1.5-4). In linear regression analysis, none of the demographic and clinical factors were found to be significantly associated with percentage change in VAS score (Table 1).

Objective measurements; Impedance-pH monitoring

The median number of SGBs was 103 (41-252) episodes. The median total number of reflux episodes was 49 (31-82) episodes, in which those of acid reflux was 37 (16-55) episodes. The median of total oesophageal acid exposure time was $3.8 \%(1.3-8.7)$ and 17 patients (44\%) had pathological acid exposure (>6\%) 23. Linear regression analysis of the impedance-pH parameters showed that only the initial number of SGBs was significantly associated with percentage change in VAS score (Table 1). Due to the strongly positively skewed distribution of this score, this variable was analysed on the log scale. Patients with a lower number of SGBs before CBT were found to have a stronger percent decrease (improvement) of the VAS score after CBT (i.e. better outcome). (Figure 1A). 
Subjective measurements; Symptom severity, health-related quality of life, personality trait, and hypervigilance

There was no significant association between percent change in VAS score from pre to post treatment and severity of VAS score at baseline or any of the initial SF-36 or Big Five Inventory-10 parameters (Table 2). However, hypervigilance score was found to be significantly associated $(P=0.04)$. Higher hypervigilance score were associated with weaker percent decrease of the VAS score (less improvement) after CBT (i.e. worse outcome) (Figure 1B).

\section{During-treatment factors associated to CBT outcome}

Out of 39 patients, 32 patients (82\%) could detect warning signals before belching. Four patients had throat symptoms, 25 patients had chest symptoms and 6 patients had epigastric symptoms as warning signals.

Neither the recognition of a warning signal or the acceptance of the behavioural character of their symptoms were significantly individually associated with better outcome. In contrast, the adherence to CBT exercises component was found to be significant. When the 3 components of the proficiency score were considered together (proficiency score), a higher score was associated with a stronger reduction of VAS score post treatment (improvement) (Figure 1C) (Table 3).

\section{Multivariate analysis using both pre- and during- CBT factors}

The number of pre-treatment SGBs, hypervigilance score and the CBT proficiency score were independently and significantly associated with the outcome. Higher values of both of the number of initial SGBs and hypervigilance 
score were associated with worse outcomes. On the contrary, higher CBT proficiency score was associated to better outcome. The $R^{2}$ value was $49 \%$ suggesting that almost half of all variation in the outcome could be explained by these three factors (Table 4)

\section{Change of VAS score along the CBT sessions}

CBT improved VAS score gradually as the number of CBT sessions increased (Figure 2). Comparing VAS score between each two consecutive sessions, significant decreases of VAS score were shown both between pre-CBT and $3^{\text {rd }}$ CBT session (261 \pm 82 vs $210 \pm 90, P<0.001)$ and between $4^{\text {th }} C B T$ session and post-CBT (182 \pm 84 vs $138 \pm 84, \mathrm{P}<0.001)$.

\section{Drop-out patients during CBT treatment}

12 patients (24\%) dropped out during CBT treatment. The average number of CBT sessions which dropouts attended was $2(1.8-3)$. Table 5 compares pretreatment and during treatment parameters between dropouts and those that completed treatment. The reasons for non-completion of treatment were lack of confidence $(n=3)$, travelling difficulties $(n=5)$ and preferred other treatments $(n=4)$. There was no significant difference in age, gender, first language, degree of education, duration of belching symptom, initial severity of symptom and quality of life between dropouts and completers. The number of initial SGBs was numerically lower in dropouts but did not reach statistical significance. Only acceptance score during CBT was significantly lower in dropouts compared to those who completed CBT. 


\section{Long-term outcome of CBT for SGB}

Thirty-one patients (79\%) completed the telephone interview at 6-12 months after CBT, of which 24 patients $(77 \%)$ stated that they continued to follow the CBT on a regular basis. Pre-CBT VAS score $(267 \pm 79)$ decreased to $151 \pm 88$ at the end of CBT $(P<0.001)$. VAS score at the $6-12$ months follow up $(153 \pm 82)$ were significantly lower than that of pre-CBT $(P<0.001)$, and no significant difference was found between that of post-CBT and follow up at 6-12 months after CBT $(P=1)$ (Figure 3). 


\section{Discussion}

Cognitive behavioural therapy is a frequently used treatment for SGB ${ }^{11-16}$. We have recently introduced CBT for SGB patients, obtaining a success rate of around $50 \%$ of our population that completed treatment. Identification of SGB patients that are more likely to respond to CBT could have significant clinical implications i.e. choice between pharmacological vs. CBT. In this study, we aimed to identify the "pre-treatment" and "during-treatment" factors affecting the outcome of CBT. Our results showed (a) that the number of initial SGBs, hypervigilance score and a CBT proficiency score were independently associated with outcome (b) CBT exercises improved symptoms gradually as the number of CBT sessions increased, (c) the effect of CBT for SGB lasted at least 6-12 months. We used as outcome measure the subjective assessment of symptoms improvement rather than objective decrease of the number of SGBs. Acquiring self-confidence in controlling SGBs is probably more relevant for patients even if the number of SGBs did not significantly diminish ${ }^{12,24}$.

Previous studies assessed the effect of CBT in patients with SGB. Hemmink et al. ${ }^{14}$ reported that $54.5 \%(6 / 11)$ of patients achieved $>30 \%$ decrease of VAS score by 10 CBT sessions. Ong et al. ${ }^{15}$ showed that 4 CBT sessions decreased VAS score by $>50 \%$ in $55.6 \%(20 / 36)$ of patients. Also, Cate et al. ${ }^{16}$ demonstrated that 5 sessions of speech therapy improved VAS score sufficiently in $60.4 \%$ $(29 / 48)$ of the patients.

In our study, a larger number of SGB and a higher score of hypervigilance were pre-treatment indicators of possible worse outcome. Similar results were suggested by studies in other functional GI disorders. A randomized control trial 
of $\mathrm{CBT}$ for functional bowel disorder demonstrated that participants with less severe illness responded better to treatment ${ }^{25}$.

- Although a relatively higher hypervigilance score was found in patients with worse response to CBT, the personality and hypervigilance scores of our sample did not differ from relevant population norms.

The proficiency score comprised three key components of CBT as follows; (a) acceptance of explanation about SGB, (b) detection of warning signals, and (c) adherence to the exercise such as diaphragmatic breathing and mouth opening manoeuvre.

The patients' acceptance of behavioural explanation was a predictor of outcome. There is a possible barrier to accept that SGB could be a learned response if the suggestion is perceived as threatening or demeaning, e.g. if it is seen as blaming the patient for bringing about their symptoms themselves, or implying that the problem is 'all in the mind'. Such perception might reduce engagement with treatment.

The finding that patients who were able to identify warning signals preceding belching benefited from treatment is novel and may have practical implications. The variable was assessed at the first treatment session and so it could not have been affected by retrospective bias or self-selection. It is possible that patients could benefit from a list of signals that the successful patients pay attention to, and from being guided to learn to detect such warning signals themselves.

The weight of adherence and acceptance was more important than the identification of the warning signal regarding the response to CBT. 
Although univariable analysis did not show significant association between detecting warning signals (isolated) and outcome, factors in the proficiency score have a profound mutual influence i.e. good acceptance and detecting warning signals can enhance patients' adherence to the therapy ${ }^{26,27}$.

A high number of initial SGBs and hypervigilance score can be detected at the time of diagnosis of pathological SGB. On the other hand, a good proficiency score during CBT was associated with good outcome. These results might suggest that patients with poor pre-treatment parameters should either be treated with Baclofen or need more intensive and frequent CBT to improve proficiency.

Our study had some limitations. Although the study included the largest number of SGB patients published so far, the sample size was still small. The analysis of parameters involved in CBT outcomes was based on our previous clinical study which lacked a control group either using pharmacotherapy (baclofen and/or pregabalin) or sham $\mathrm{CBT}^{8}$. However, a recent randomised control trial comparing the effect of CBT versus no treatment on patients with SGBs did not show significant improvement in the no treatment group ${ }^{15}$.

We could not fully evaluate outcomes in dropout's patients. The proficiency score might be biased because the psychologists rated it at the end of CBT where the therapist knew whether the patient improved or not; and the ratings were provided by the therapist, rather than by an independent observer. However, ratings should be implemented only at the end of treatment because the patients' proficiency for CBT got better by the increment of the number of CBT sessions 
they attended. Lastly, long-term follow-up did not examine the number of SGBs using impedance-pH monitoring at 6-12 month after CBT.

In conclusion, the number of SGBs, hypervigilance score and CBT proficiency for SGB were factors that influenced treatment outcome. This result suggested that clinicians could detect patients with SGB who are less likely to respond to CBT at diagnosis. Based on this information, it is possible to modify the CBT intensity and duration to improve outcome. 


\section{References}

1. Bredenoord AJ, Weusten BL, Sifrim D, Timmer R, Smout AJ. Aerophagia, gastric, and supragastric belching: a study using intraluminal electrical impedance monitoring. Gut. 2004;53(11):1561-1565.

2. Koukias N, Woodland P, Yazaki E, Sifrim D. Supragastric Belching: Prevalence and Association With Gastroesophageal Reflux Disease and Esophageal Hypomotility. J Neurogastroenterol Motil. 2015;21(3):398-403.

3. Kessing BF, Bredenoord AJ, Velosa M, Smout AJ. Supragastric belches are the main determinants of troublesome belching symptoms in patients with gastro-oesophageal reflux disease. Aliment Pharmacol Ther. 2012;35(9):1073-1079.

4. Hemmink GJ, Bredenoord AJ, Weusten BL, Timmer R, Smout AJ. Supragastric belching in patients with reflux symptoms. $A m \mathrm{~J}$ Gastroenterol. 2009;104(8):1992-1997.

5. Piessevaux H, De Winter B, Louis E, et al. Dyspeptic symptoms in the general population: a factor and cluster analysis of symptom groupings. Neurogastroenterol Motil. 2009;21(4):378-388.

6. Obekli T, Akyuz F, Akyuz U, et al. Belching in Irritable Bowel Syndrome: An Impedance Study. J Neurogastroenterol Motil. 2017;23(3):409-414.

7. Bredenoord AJ, Smout AJ. Impaired health-related quality of life in patients with excessive supragastric belching. Eur J Gastroenterol Hepatol. 2010;22(12):1420-1423.

8. Glasinovic E, Wynter E, Arguero J, et al. Treatment of supragastric belching with cognitive behavioral therapy improves quality of life and reduces acid gastroesophageal reflux. Am J Gastroenterol. 2018;113(4):539-547.

9. Kunte H, Kronenberg G, Fink K, Harms L, Hellweg R. Successful treatment of excessive supragastric belching by combination of pregabalin and baclofen. Psychiatry Clin Neurosci. 2015;69(2):124-125.

10. Blondeau K, Boecxstaens V, Rommel N, et al. Baclofen improves symptoms and reduces postprandial flow events in patients with rumination and supragastric belching. Clin Gastroenterol Hepatol. 2012;10(4):379-384.

11. Katzka DA. Simple office-based behavioral approach to patients with chronic belching. Dis Esophagus. 2013;26(6):570-573. 
12. Riehl ME, Kinsinger S, Kahrilas PJ, Pandolfino JE, Keefer L. Role of a health psychologist in the management of functional esophageal complaints. Dis Esophagus. 2015;28(5):428-436.

13. Cigrang JA, Hunter CM, Peterson AL. Behavioral treatment of chronic belching due to aerophagia in a normal adult. Behav Modif. 2006;30(3):341-351.

14. Hemmink GJ, Ten Cate L, Bredenoord AJ, Timmer R, Weusten BL, Smout AJ. Speech therapy in patients with excessive supragastric belching--a pilot study. Neurogastroenterol Motil. 2010;22(1):24-28, e22-23.

15. Ong AM, Chua LT, Khor CJ, Asokkumar R, S/O Namasivayam V, Wang YT. Diaphragmatic Breathing Reduces Belching and Proton Pump Inhibitor Refractory Gastroesophageal Reflux Symptoms. Clin Gastroenterol Hepatol. 2018;16(3):407-416.e402.

16. Ten Cate L, Herregods TVK, Dejonckere PH, Hemmink GJM, Smout AJPM, Bredenoord AJ. Speech Therapy as Treatment for Supragastric Belching. Dysphagia. 2018;33(5):707-715.

17. Bowling A, Bond M, Jenkinson C, Lamping DL. Short Form 36 (SF-36) Health Survey questionnaire: which normative data should be used? Comparisons between the norms provided by the Omnibus Survey in Britain, the Health Survey for England and the Oxford Healthy Life Survey. J Public Health Med. 1999;21(3):255-270.

18. Rammstedt B , John OP. Measuring personality in one minute or less: a 10-item short version of the Big Five Inventory in English and German. J Res Pers 2007;41:203- 12.

19. Barsky AJ, Wyshak G, Klerman GL. The somatosensory amplification scale and its relationship to hypochondriasis. J Psychiatr Res. 1990;24(4):323-334.

20. Bridou M, Aguerre C. Validity of the French form of the Somatosensory Amplification Scale in a Non-Clinical Sample. Health Psychol Res. 2013;1(1):e11.

21. Nakao M, Barsky AJ, Kumano H, Kuboki T. Relationship between somatosensory amplification and alexithymia in a Japanese psychosomatic clinic. Psychosomatics. 2002;43(1):55-60.

22. Nakao M, Barsky AJ. Clinical application of somatosensory amplification in psychosomatic medicine. Biopsychosoc Med. 2007;1:17.

23. Gyawali CP, Kahrilas PJ, Savarino E, et al. Modern diagnosis of GERD: 
the Lyon Consensus. Gut. 2018;67(7):1351-1362.

24. Levy RL, Olden KW, Naliboff BD, et al. Psychosocial aspects of the functional gastrointestinal disorders. Gastroenterology. 2006;130(5):14471458.

25. Drossman DA, Toner BB, Whitehead WE, et al. Cognitive-behavioral therapy versus education and desipramine versus placebo for moderate to severe functional bowel disorders. Gastroenterology. 2003;125(1):1931.

26. Ballou S, Keefer L. Psychological Interventions for Irritable Bowel Syndrome and Inflammatory Bowel Diseases. Clin Trans/ Gastroenterol. 2017;8(1):e214.

27. Matsuzawa $Y$, Lee YSC, Fraser F, et al. Barriers to Behavioral Treatment Adherence for Headache: An Examination of Attitudes, Beliefs, and Psychiatric Factors. Headache. 2018. 
Table 1 Univariable analysis of objective pre-CBT factors such as demographic and clinical characteristics and impedance-pH measurements

\begin{tabular}{|c|c|c|}
\hline Variable & Coefficient $(95 \% \mathrm{CI})$ & P-value \\
\hline Age $(\text { years })^{+}$ & $5(-3,12)$ & 0.20 \\
\hline Female & $-12(-32,9)$ & 0.26 \\
\hline Native English speaker & $-10(-32,11)$ & 0.33 \\
\hline Education to degree or above & $-5(-30,20)$ & 0.71 \\
\hline Primary symptom (belch) & $-15(36,6)$ & 0.16 \\
\hline Duration of belching symptom (years) & $0(-3,3)$ & 0.92 \\
\hline \multicolumn{3}{|l|}{ impedance-pH measurements } \\
\hline Number of SGBs $\ddagger$ & $25(7,41)$ & 0.006 \\
\hline Total reflux $\S$ & $1(-2,4)$ & 0.62 \\
\hline Acid reflux $\S$ & $3(-1,6)$ & 0.19 \\
\hline Non-acid reflux $\S$ & $-2(-7,3)$ & 0.41 \\
\hline Total acid exposure time (\%) & $3(-9,14)$ & 0.64 \\
\hline Upright acid exposure time (\%) $\pi$ & $4(-5,12)$ & 0.37 \\
\hline Recumbent acid exposure time $(\%) \pi$ & $-2(-11,7)$ & 0.68 \\
\hline
\end{tabular}

+ Regression coefficient given for a 10 -year increase in age

$\ddagger$ Variable analysed on the log scale (base 10)

$\S$ Regression coefficients given for a 10 -unit increase in variable

$\pi$ Regression coefficients given for a 5-unit increase in variable

CBT, cognitive behaviour therapy; SGBs, supragastric belchings; CI, confidence interval. 
Table 2 Univariable analysis of subjective pre-CBT factors such as symptom severity, quality of life, personality trait, and hypervigilance

\begin{tabular}{|c|c|c|}
\hline Variable & Coefficient $(95 \% \mathrm{CI})$ & P-value \\
\hline VAS score at baseline $^{\dagger}$ & $-1(-8,5)$ & 0.65 \\
\hline \multicolumn{3}{|l|}{ SF-36 } \\
\hline Physical functioning $\ddagger$ & $-1(-4,3)$ & 0.68 \\
\hline Role limitation physical $\ddagger$ & $-1(-4,1)$ & 0.29 \\
\hline Bodily pain $\ddagger$ & $0(-4,3)$ & 0.92 \\
\hline General health perceptions $\ddagger$ & $1(-4,6)$ & 0.71 \\
\hline Vitality $\ddagger$ & $2(-3,6)$ & 0.49 \\
\hline Social functioning $\ddagger$ & $-2(-6,2)$ & 0.22 \\
\hline Role-emotional ‡ & $-2(-4,1)$ & 0.13 \\
\hline Mental health $\ddagger$ & $-1(-5,4)$ & 0.79 \\
\hline \multicolumn{3}{|l|}{ Big Five Inventory-10 } \\
\hline Extraversion & $2(-10,14)$ & 0.75 \\
\hline Agreeableness & $-7(-21,8)$ & 0.37 \\
\hline Conscientiousness & $-7(-21,8)$ & 0.38 \\
\hline Neuroticism & $-7(-16,2)$ & 0.13 \\
\hline Openness & $-4(-16,9)$ & 0.53 \\
\hline Hypervigilance score (SSAS)§ & $7(0,13)$ & 0.04 \\
\hline
\end{tabular}

${ }^{\dagger}$ Regression coefficients given for a 50 -unit increase in variable

${ }^{\ddagger}$ Regression coefficients given for a 10 -unit increase in variable

$\S$ Regression coefficients given for a 5-unit increase in variable

CBT, cognitive behaviour therapy; VAS, Visual analogue scale; SF-36, the Short Form Health Survey; SSAS, Somatosensory Amplification Scale; CI, confidence interval. 
Table 3 Univariable analyses of during CBT factors

\begin{tabular}{lcc}
\hline Variable & Coefficient (95\% CI) & P-value \\
\hline Individual CBT components & $-17(-44,10)$ & 0.22 \\
$\quad$ Warning signal & $-18(-38,3)$ & 0.09 \\
Acceptance (Score 2) & $-34(-6,-13)$ & 0.002 \\
Adherence (Score 2) & & \\
& $-11(-17,-5)$ & 0.001 \\
Proficiency score for CBT (continuous) & 0.01 \\
Proficiency score for CBT (categorical) & $-28(-54,-1)$ & \\
Score 0 - 2 & $-38(-62,-13)$ & \\
Score 3-4 & & \\
Score 5 & & \\
\hline
\end{tabular}

CBT, cognitive behaviour therapy; CI, confidence interval. 
Table 4 Multivariable model

\begin{tabular}{lccc}
\hline Variable & Coefficient (95\% CI) & P-value & Model R $^{2}$ \\
\hline Number of SGBs ${ }^{\dagger}$ & $25(10,40)$ & 0.002 & $49 \%$ \\
Hypervigilance score (SSAS) $^{\ddagger}$ & $7(2,13)$ & 0.01 & \\
Proficiency score for CBT & $-8(-13,-2)$ & 0.008 & \\
\hline
\end{tabular}

$\dagger$ Variable analysed on the log scale (base 10)

‡ Regression coefficients given for a 5-unit increase in variable

SGBs, supragastric belchings; SSAS, Somatosensory Amplification Scale; CBT, cognitive behaviour therapy; CI, confidence interval. 
Table 5 Demographic and clinical characteristics, quality of life and during-CBT factors in patients who completed and dropped out of CBT.

\begin{tabular}{|c|c|c|c|}
\hline & $\begin{array}{c}\text { Subjects who } \\
\text { completed CBT } \\
\mathrm{N}=39\end{array}$ & $\begin{array}{l}\text { Dropouts } \\
\mathrm{N}=12\end{array}$ & P-value \\
\hline Age (years) & $46(32-56)$ & $47(35-51)$ & 0.97 \\
\hline Female $(\%)$ & $20(51.3)$ & $4(33.3)$ & 0.34 \\
\hline Native English speaker (\%) & $23(59.0)$ & $8(66.7)$ & 0.74 \\
\hline Education to degree or above (\%) & $9(23.1)$ & $3(25.0)$ & 1 \\
\hline $\begin{array}{l}\text { Duration of belching symptom } \\
\text { (years) }\end{array}$ & $2.5(1.5-4)$ & $2.8(2-3.5)$ & 0.57 \\
\hline Number of SGBs & $114(44-228)$ & $55(33-76)$ & 0.07 \\
\hline VAS score at baseline & $6.5(5.4-8)$ & $7.8(6.5-9)(\mathrm{N}=9)$ & 0.20 \\
\hline \multicolumn{4}{|l|}{ SF-36 } \\
\hline Physical functioning & $65(35-89)$ & $80(64-96)$ & 0.23 \\
\hline Role limitation physical & $50(0-100)$ & $38(0-75)$ & 0.42 \\
\hline Bodily pain & $45(24-78)$ & $68(30-78)$ & 0.71 \\
\hline General health perceptions & $45(30-60)$ & $43(19-58)$ & 0.56 \\
\hline Vitality & $50(28-58)$ & $55(44-60)$ & 0.45 \\
\hline Social functioning & $63(38-75)$ & $63(38-75)$ & 0.96 \\
\hline Role-emotional & $100(33-100)$ & $67(50-100)$ & 0.56 \\
\hline Mental health & $64(48-80)$ & $62(54-68)$ & 0.71 \\
\hline \multicolumn{4}{|l|}{ During-CBT factors } \\
\hline Warning signal (\%) & $32(82.1)$ & $7(58.3)$ & 0.12 \\
\hline Acceptance & $1(1-2)$ & $0(0-1)$ & 0.004 \\
\hline
\end{tabular}

CBT, cognitive behaviour therapy; SGBs, supragastric belchings; VAS, Visual analogue scale; SF-36, the Short Form Health Survey. 
Figure legends

Figure 1 Association between number of SGBs, Hypervigilance score, and CBT proficiency score and percent change in VAS score

Note that in the graph the number of SGBs is shown on the log scale (A). The fitted regression lines are represented as red line. High number of SGBs (A) and hypervigilance score (SSAS) (B) were associated with large percent change of VAS score (i.e. worse outcome). On the contrary, high total proficiency score (on a continuous scale) was related to smaller percent change of VAS score (i.e. better outcome) (C).

SGB, supragastric belching; SSAS, Somatosensory Amplification Scale; CBT, cognitive behavioral therapy; VAS, Visual analogue scale.

Figure 2 Shift of VAS score from baseline during the CBT sessions

VAS score gradually decreased as the CBT sessions progressed. VAS score at 3rd session was significantly smaller than those at Pre-CBT. Similarly, post-CBT showed significantly smaller VAS score compared to $4^{\text {th }}$ CBT session.

VAS, Visual analogue scale; CBT, cognitive behavioral therapy.

${ }^{*} p<0.001$

Figure 3 Long-term outcome of CBT for SGB

CBT significantly improved VAS score not only at just after the treatment sessions. This effect lasted 6 to 12 months after the treatment as no significant change was observed between just post-CBT and follow-up at 6-12months after CBT.

$\mathrm{CBT}$, cognitive behavioral therapy; SGB, supragastric belching; VAS, Visual analogue scale.

${ }^{*} p<0.001$ versus pre-CBT 